CLINICAL STUDY

\title{
Presence of pituitary adenylate cyclase activating polypeptide- 38 in human plasma and milk
}

\author{
Rita Borzsei $^{1, *}$, Laszlo Mark ${ }^{2, *}$, Andrea Tamas $^{3}$, Terez Bagoly ${ }^{1}$, Csaba Bay ${ }^{4}$, Katalin Csanaky $^{3}$, Eszter Banki $^{3}$, \\ Peter Kiss ${ }^{3}$, Alexandra Vaczy ${ }^{2}$, Gabriella Horvath ${ }^{3}$, Jozsef Nemeth ${ }^{5}$, Edit Szauer ${ }^{3}$, Zsuzsanna Helyes ${ }^{1, *}$ \\ and Dora Reglodi ${ }^{3, *}$ \\ Departments of ${ }^{1}$ Pharmacology and Pharmacotherapeutics, ${ }^{2}$ Biochemistry and Medical Chemistry, ${ }^{3}$ Anatomy, ${ }^{4}$ Obstetrics and Gynaecology, University of \\ Pecs, Szigeti u 12, 7624 Pecs, Hungary and ${ }^{5}$ Department of Pharmacology and Pharmacotherapeutics, University of Debrecen, 4010 Debrecen, Hungary \\ (Correspondence should be addressed to D Reglodi; Email: dora.reglodi@aok.pte.hu)
}

*(R Borzsei and L Mark, as well as Z Helyes and D Reglodi made equal contributions to this work)

\begin{abstract}
Objective: Pituitary adenylate cyclase activating polypeptide (PACAP) is a pleiotropic and multifunctional neuropeptide widely distributed throughout the body. It is involved in the regulation of various physiological and pathophysiological processes, such as reproduction, thermoregulation, motor activity, brain development, neuronal survival, inflammation and pain. Since little is known about its distribution in humans, our aim was to examine PACAP-38 in human plasma. Furthermore, based on the presence of vasoactive intestinal peptide, structurally the closest to PACAP, in milk and PACAP and its receptors in the mammary gland, our aim was to study PACAP-38 in human milk. Design and methods: The presence of PACAP-38 was determined by mass spectrometry in plasma samples from healthy male and female volunteers (age: 20-40), as well as in plasma and milk samples from lactating women (age: 20-35). PACAP concentration was measured with a specific and sensitive RIA.

Results: Our results revealed that PACAP-38 is present in human plasma, its concentration is relatively stable in healthy volunteers and it is not significantly altered by gender, age, food intake or hormonal cycle in females. However, PACAP-38 plasma levels significantly increased in lactating women having 1-6 month-old babies. Moreover, this study is the first which provides evidence for the presence of PACAP-38 in the human milk with levels 5-20-fold greater in the milk whey than in the respective plasma samples.

Conclusions: We found PACAP-38 in human plasma and its increase during the first 6 months of the lactation period. A prominent, nearly 10-fold higher concentration of this peptide was detected in human milk. Based on the literature, several important actions of milk-derived PACAP-38 can be suggested such as mammary gland proliferation, nutrient transfer as well as regulation of growth/differentiation of certain tissues of the neonates. The novelty of the present descriptive data provides a basis for further investigations on the mechanism of PACAP-38 secretion in human milk and its functional significance.
\end{abstract}

European Journal of Endocrinology 160 561-565

\section{Introduction}

Pituitary adenylate cyclase activating polypeptide (PACAP) belongs to the vasoactive intestinal peptide (VIP)/secretin/glucagon peptide family. It was isolated from the hypothalamus as an activator of adenylate cyclase in the pituitary gland. However, since its discovery, PACAP has proved to be a pleiotropic and multifunctional neuropeptide (1). It is found in two forms, PACAP-27 and PACAP-38, with PACAP-38 being the major form (1). PACAP is widely distributed in the nervous system and also in endocrine glands, cardiovascular, gastrointestinal and respiratory tracts. It is involved in the regulation of various physiological processes, such as feeding, reproduction, thermoregulation, catecholamine synthesis, motor activity, brain development and neuronal survival $(1,2)$. Little is known about the functions of PACAP in humans. Intravenous infusion of PACAP increases ACTH, vasopressin and prolactin levels (3). It also increases heart rate and slightly decreases cerebral blood flow (4).

PACAP has been found in rat and human blood. RIA analysis of rat serum has revealed that PACAP-38 concentration is higher in the hypophyseal portal blood than in peripheral blood (5). We have previously identified PACAP-38 in rat plasma by mass spectrometry and provided evidence that PACAP is released from activated capsaicin-sensitive afferents into the 
circulation in rats, using RIA analysis $(6,7)$. In the first part of our study, we investigated the presence and concentration of PACAP-38 in human plasma of healthy male and female volunteers and in lactating women.

Human milk contains a variety of proteins and peptides possessing biological activity. Several hormones have been described in the milk, including pituitary, hypothalamic, pancreatic, thyroid, adrenal, gonadal, gut hormones and growth factors such as insulin-like, epidermal, transforming, and nerve growth factors (8). High concentrations of VIP, structurally the closest to PACAP, have been measured in milk (9). Although PACAP-immunoreactive fibres have been shown in the mammary gland of rats (10) and all 3 PACAP receptors (PAC1, VPAC1 and 2) have been identified in normal and cancerous human mammary glands (11), there are no data regarding the presence and concentration of PACAP in human milk. Therefore, we investigated the presence of PACAP in human milk and its correlation with plasma levels.

\section{Methods}

Human blood ( $10 \mathrm{ml} /$ person) was collected from cubital veins of healthy volunteers of both sexes (age between 20 and $40 ; n=19)$ and healthy lactating women who had 1-6 month-old babies (age between 20 and 35 years; $n=31$ ) according to a protocol approved by the institutional ethic committee into ice-cold glass tubes containing EDTA $(18 \mathrm{mg})$ and the peptidase inhibitor aprotinin (1200 U). Samples were centrifuged at 1000 r.p.m. for $10 \mathrm{~min}$ and 4000 r.p.m. for $10 \mathrm{~min}$ at $4{ }^{\circ} \mathrm{C}$. Human milk ( $5 \mathrm{ml} /$ person) was also obtained from healthy lactating women into ice-cold tubes.

For mass spectrometry, $1 \mathrm{ml}$ human plasma was filtered on a centrifugal membrane filter device (Amicon Ultra-4 10000 MWCO, Millipore Kft., Budapest, Hungary). The ultrafiltration devices were centrifuged in a swinging bucket rotor for $30 \mathrm{~min}$ at $3000 \mathrm{~g}$. The filtrate was liophylized and redissolved in $200 \mu \mathrm{l}$ of $0.1 \%$ trifluoro-acetic acid (TFA). The solution was desalted and cleaned using $0.1 \%$ TFA and acetonitrile/0.1\% TFA $(2 / 98, v / v)$ solutions with ZipTip 18 pipette tips (Millipore $\mathrm{Kft}$ ). Elution was carried out by acetonitrile/0.1\% TFA $(50 / 50, v / v)$ solution. The lipid fraction of the milk sample was precipitated by chilling $\left(0-4{ }^{\circ} \mathrm{C}\right)$ and the liquid phase of the mixture was loaded to MALDI target plate (MTP 384 massive target T, Bruker Daltonics, Bremen, Germany) without any further sample preparation.

Identification of PACAP-38 was performed with matrix-assisted laser desorption/ionization time-offlight (MALDI TOF/TOF) mass spectrometry, as described previously (6). Briefly, the mass spectrometer used in this work was an Autoflex II TOF/TOF (Bruker Daltonics) operated in the linear detector for MALDI
TOF or LIFT mode for post source decay MALDI TOF/TOF with an automated mode using the FlexControl software. The aqueous solutions of the PACAP-38 standard (Sigma-Aldrich Ltd) and the human serum and milk samples were loaded onto the target plate (MTP 384 massive target T, Bruker Daltonics) by mixing $1 \mu \mathrm{l}$ of each solution with the same volume of a saturated matrix solution, prepared fresh every day by dissolving $\alpha$-cyano-4-hydroxycinnamic acid in acetonitrile/0.1\% TFA $(1 / 2, \mathrm{v} / \mathrm{v})$. The ions were accelerated under delayed extraction conditions (200 ns) in positive ion mode with an acceleration voltage of $20.00 \mathrm{kV}$. The instrument uses a $337 \mathrm{~nm}$ pulsed nitrogen laser, model MNL-205MC (LTB Lasertechnik Berlin GmbH., Berlin, Germany). External calibration was performed in each case using Bruker Peptide Calibration Standard (\#206195 Peptide Calibration Standard, Bruker Daltonics). Protein masses were acquired with a range of $\mathrm{m} / \mathrm{z} 1000$ to m/z 10 000. Each spectrum was produced by accumulating data from 200 consecutive laser shots for standard solution and 1000 for milk and serum samples. The Bruker FlexControl 2.4 software was used for control of the instrument and the Bruker FlexAnalysis 2.4 software for spectra evaluation.

PACAP-38-like immunoreactivity in the human plasma and milk whey was determined with a specific and sensitive RIA technique developed in our laboratory $(6,7)$ and concentrations of the peptide were calculated with a calibration curve. The peptide was extracted from $3 \mathrm{ml}$ plasma samples by addition of a double volume of absolute alcohol and $20 \mu \mathrm{l} \mathrm{96 \%} \mathrm{acetic} \mathrm{acid.}$ After precipitation and a second centrifugation (3000 r.p.m. for $20 \mathrm{~min}$ at $4{ }^{\circ} \mathrm{C}$ ) the samples were dried under nitrogen flow and resuspended in $300 \mu \mathrm{l}$ assay buffer to achieve a concentration 10-fold higher for the RIA procedure $(6,7)$. For RIA analysis of milk, $10 \mu \mathrm{l} \mathrm{96 \%} \mathrm{acetic} \mathrm{acid} \mathrm{was} \mathrm{added} \mathrm{to} 1 \mathrm{ml}$ milk and incubated in $40{ }^{\circ} \mathrm{C}$ water bath for 5 min to precipitate the protein content. Centrifugation was performed at 4000 r.p.m. for $10 \mathrm{~min}$ at $4{ }^{\circ} \mathrm{C}$ to obtain a solid fat component on the top of the samples. The whey localized between the precipitated protein and fat fractions was then removed for RIA analysis. RIA analysis using the '88111-3' PACAP-38 antiserum was performed as previously described $(6,7)$. PACAP-38 concentrations of the samples were read from a calibration curve created using the standard preparations.

\section{Results}

The protonated quasimolecular ion was detected at $\mathrm{m} / \mathrm{z} 4535$ in the PACAP standard (Fig. 1A). The post source decay MALDI TOF/TOF fragmentation of PACAP-38 standard yielded mainly y fragment ions of the PACAP-38 parent ion (4535 kDa; Fig. 1B). Moreover, the developed sample preparation enabled 
A
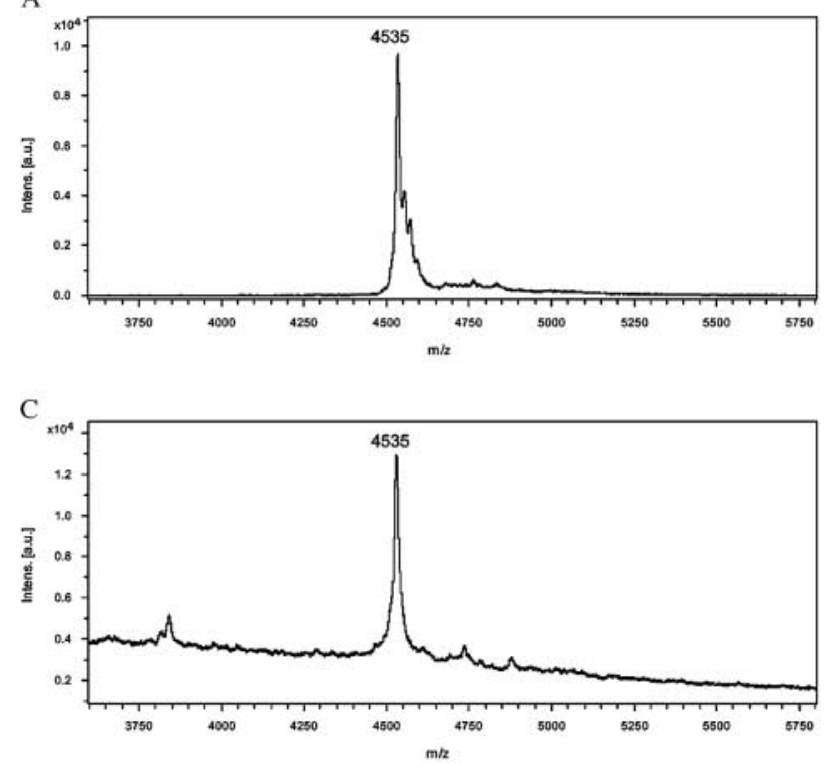

E

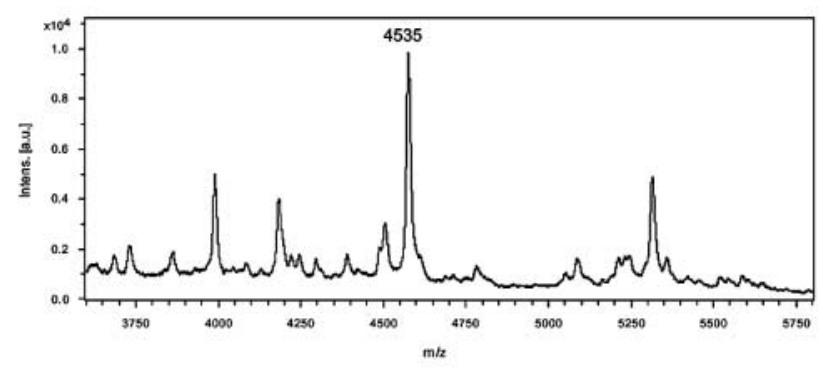

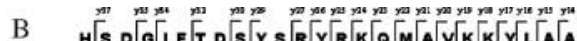
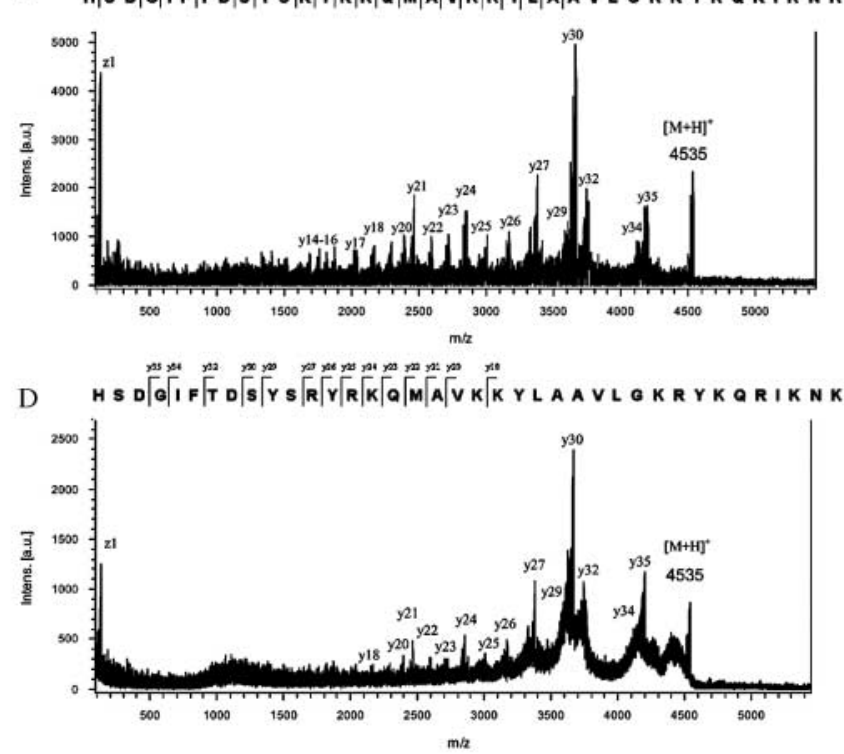

F

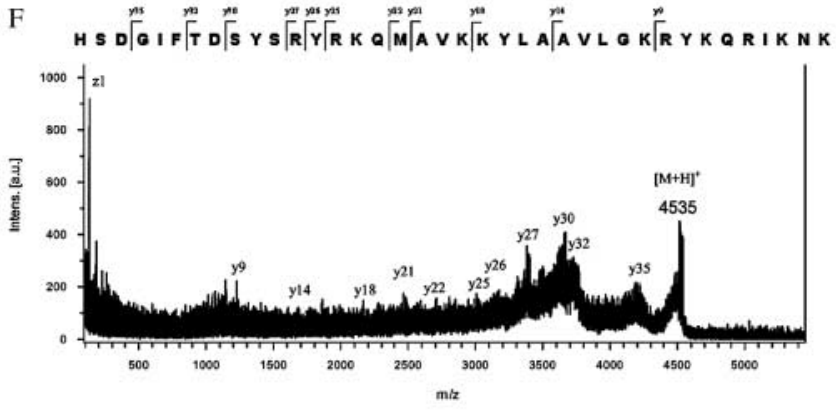

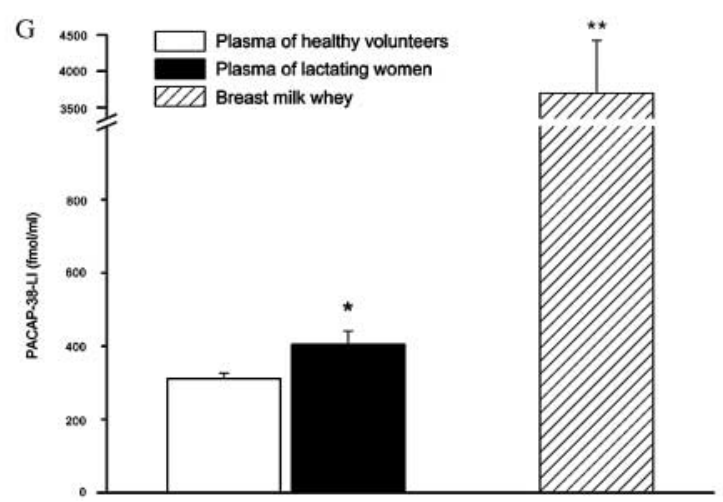

Figure 1 MALDI TOF spectrum of PACAP-38 in positive ion mode using linear detection indicating the protonated quasimolecular ion [M+ $\mathrm{H}^{+}$] of PACAP38 in (A) standard (C) human serum and (E) milk. Post source decay fragmentation of PACAP-38 of the $4535 \mathrm{~m} / \mathrm{z}$ peak as a parent ion by using LIFT mode with the amino acid sequence in (B) standard, (D) human serum (F) and human milk. (G) PACAP-38-like immunoreactivity determined by RIA from the human plasma and breast milk whey. ${ }^{*} P<0.05$ versus plasma of healthy volunteers; ${ }^{* *} P<0.001$ versus respective plasma.

us to detect the quasimolecular ion of PACAP-38 in human serum (Fig. 1C). The results of mass spectrometry could unequivocally prove the presence of PACAP-38 in serum samples. The identity of the post source fragmentation standard and that of the serum samples provided a further piece of evidence for the presence of PACAP-38 in human blood (Fig. 1D). Similarly to serum samples, the peak characteristic for PACAP-38 could be detected in milk samples (Fig. 1E). The post source decay fragmentation also showed the fragments identical to standard and serum PACAP-38 fragments (Fig. 1F). 
PACAP-38 could be accurately and reliably measured in the human plasma with relatively small interindividual differences among healthy volunteers (both sexes, age between 20 and 40 years) (Fig. 1G). Age, gender or the hormonal cycle in females (data not shown) had no significant influence on plasma PACAP38. However during lactation, the plasma concentration of this peptide moderately, but significantly increased compared with the plasma level of healthy volunteers (Fig. 1G). Similarly to the plasma, this RIA technique also proved to be very sensitive and specific for the determination of PACAP-38-like immunoreactivity in the milk. Surprisingly, the concentration of this peptide was 5-20-fold higher in the milk whey than in the respective plasma samples (Fig. 1G). Neither the duration of the lactation (age of the infants) nor the frequency of breast-feeding altered the concentration of this peptide within the 1-6 month-period (data not shown).

\section{Discussion}

Our results revealed that PACAP-38 is present in human plasma and its concentration, which can be reliably measured with RIA, is relatively stable in 20-40 year-old healthy volunteers. It is not significantly altered by gender, age, food intake or hormonal cycle in females. However, PACAP-38 plasma levels moderately, but significantly increased in lactating women having 1-6 month-old babies. The exact source of PACAP in the plasma is not known, it is most likely to be derived from neural and endocrine elements. We have recently shown the elevation of plasma PACAP-38-like immunoreactivity in rats by chemically stimulating sensory nerve endings throughout the body (6). The relatively high concentration of this peptide might be due to the prevention of its otherwise rapid degradation (2) by the addition of the peptidase inhibitor aprotinin. Moreover, this study is the first which provides evidence for the presence and high concentration of PACAP-38 in the human milk; its levels are 5-20-fold greater in the milk whey than in the respective plasma samples.

Milk contains several gastrointestinal neuropeptides, like gastric inhibitory peptide, bombesin, peptide YY, neurotensin, gastrin, cholecystokinin and peptide histidine methionine and various growth factors $(8,12)$. Several growth factors are present in higher concentrations in the milk than in the plasma, such as GnRH, TRH, VIP, somatostatin, GHRH, relaxin, insulinlike growth factor-1, epidermal growth factor, transforming growth factor and prostaglandins $(8,12)$. These bioactive compounds may play a role in the growth of the mammary gland and function in nutrient transfer and regulation of growth and differentiation of neonatal tissues.

The role of PACAP in milk can only be hypothesized at the moment. Being present at such high concentrations suggests that PACAP is required for the growth and development of the newborn. PACAP has been shown to play very important roles during the development of various organs, especially the nervous system $(1,2)$. PACAP functions as a neurotrophic factor from very early stages of neuronal development influencing neurogenesis, differentiation and patterning, and it is also involved in processes that continue after birth, such as astrocytogenesis, myelination, cerebellar development and neuronal migration. Multifunctional milk components protect against several pathogens and also stimulate the development of the natural defensive mechanisms $(8,12)$. PACAP has a variety of immunomodulatory actions, both during the development of the immune system and in mature lymphocytes and macrophages $(1,2)$. The role of endogenous PACAP is further supported by knockout studies, describing several morphological and functional alterations in mice (13).

Another possible function could be the regulation of growth and function of the mammary gland itself. PACAP is well-known for its antiapoptotic actions and its involvement in regulating cell cycle $(1,2)$. Apoptosis plays a key role also in the development of the mammary gland (14). Influenced by growth factors, the expression of antiapoptotic molecules is high during lactation, while the involuting mammary gland undergoes massive cell loss by apoptosis.

A further important question is the oral bioavailability of PACAP derived from the milk. PACAP is cleaved by dipeptidyl peptidase IV, which is the major factor limiting the half-life of biologically active peptides (15). It has been shown that the half life of PACAP and related peptides is relatively short (minutes) in body fluids (15). The mammary gland produces protease inhibitors that are responsible for the stability of various milk-borne proteins and peptides. Neonates possess lower proteolytic activity, including immaturity of the dipeptidyl peptidase IV, and have a higher permeability for macromolecules allowing significant absorption of proteins/peptides through the intestinal epithelium (8).

In conclusion, we have described the presence of PACAP-38 in the human plasma and its increase during the first 6 months of the lactation period. Furthermore, a nearly 10-fold concentration of this peptide was detected in the human milk. Although the literature suggests several important actions of milk-derived PACAP-38, such as mammary gland proliferation, nutrient transfer as well as regulation of growth/differentiation of certain tissues of the infants, these interesting and novel data are descriptive and the mechanism of PACAP-38 production/secretion and its functional significance needs further investigation.

\section{Declaration of interest}

The authors declare that there is no conflict of interest that could be perceived as prejudicing the impartiality of the research reported. 


\section{Funding}

The present work was supported by OTKA K72592, K73044, F67830, 78480, K75965, ETT439/2006 and Janos Bolyai Postdoctoral Research Fellowship.

\section{Acknowledgements}

The authors thank the help of Bea Szalontai, Attila Gaspar and all the volunteers.

\section{References}

1 Vaudry D, Gonzalez BJ, Basille M, Yon L, Fournier A \& Vaudry H. Pituitary adenylate cyclase-activating polypeptide and its receptors: from structure to functions. Pharmacological Reviews $2000 \mathbf{5 2}$ 269-324.

2 Somogyvari-Vigh A \& Reglodi D. Pituitary adenylate cyclase activating polypeptide: a potential neuroprotective peptide. Current Pharmaceutical Design 200410 2861-2889. Review.

3 Chiodera P, Volpi R, Capretti L, Caffarri G, Magotti MG \& Coiro V. Effects of intravenously infused pituitary adenylate cyclaseactivating polypeptide on adenohypophyseal hormone secretion in normal men. Neuroendocrinology $1996 \mathbf{6 4} 242-246$.

4 Birk S, Sitarz JT, Petersen KA, Oturai PS, Kruuse C, Fahrenkrug J \& Olesen J. The effect of intravenous PACAP38 on cerebral hemodynamics in healthy volunteers. Regulatory Peptides 2007 140 185-191.

5 Dow RC, Beenie J \& Fink G. Pituitary adenylate cyclase activating peptide-38 (PACAP-38) is released into hypophyseal portal blood in the normal male and female rat. The Journal of Endocrinology 1994142 R1-R4.
6 Helyes Z, Pozsgai G, Borzsei R, Nemeth J, Bagoly T, Mark L, Pinter E, Toth G, Elekes K, Szolcsanyi J \& Reglodi D. Inhibitory effect of PACAP38 on acute neurogenic and non-neurogenic inflammation in the rat. Peptides $2007 \mathbf{2 8} 1847-1855$.

7 Nemeth J, Reglodi D, Pozsgai G, Szabo A, Elekes K, Pinter E, Szolcsanyi J \& Helyes ZS. Effect of PACAP-38 on sensory neuropeptide release and neurogenic inflammation in rats and mice. Neuroscience $2006143223-230$.

8 Grosvernor CE, Picciano MF \& Baumrucker CR. Hormones and growth factors in milk. Endocrine Reviews 199214 710-728.

9 Werner H, Koch Y, Fridkin M, Fahrenkrug J \& Gozes I. High levels of vasoactive intestinal peptide in human milk. Biochemical and Biophysical Research Communications $1985133228-232$.

10 Skakkebaek M, Hannibal J \& Fahrenkrug J. Pituitary adenylate cyclase activating polypeptide (PACAP) in the rat mammary gland. Cell Tissue Research 1999298 153-159.

11 Garcia-Fernandez MO, Collado B, Bodega G, Cortes J, RuizVillaespesa A, Carmena MJ \& Prieto JC. Pituitary adenylate cyclase activating peptide/vasoactive intestinal peptide receptors in human normal mammary gland and breast cancer tissue. Gynecological Endocrinology 200520 327-333.

12 Koldovsky $\mathrm{O}$. The potential physiological significance of milkborne hormonally active substances for the neonate. Journal of Mammary Gland Biology and Neoplasia 19961 317-323.

13 Sherwood NM, Adams BA, Isaac ER, Wu S \& Fradinger EA. Knocked down and out: PACAP in development, reproduction and feeding. Peptides 200728 1680-1687.

14 Green KA \& Streuli CH. Apoptosis regulation in the mammary gland. Cellular and Molecular Life Sciences 200461 1867-1883.

15 Bourgault S, Vaudry D, Botia B, Couvineau A, Laburthe M, Vaudry H \& Fournier A. Novel stable PACAP analogs with potent activity towards the PAC1 receptor. Peptides 200829 919-932.

Received 9 January 2009

Accepted 22 January 2009 